\title{
Prevalência de vulvovaginites em mulheres da zona rural
}

\author{
Prevalence of vulvovaginite in women rural \\ Prevalencia de vulvovaginitis en mujeres del área rural
}

Recebido: 26/01/2022 | Revisado: 30/01/2022 |Aceito: 05/02/2022 | Publicado: 09/02/2022

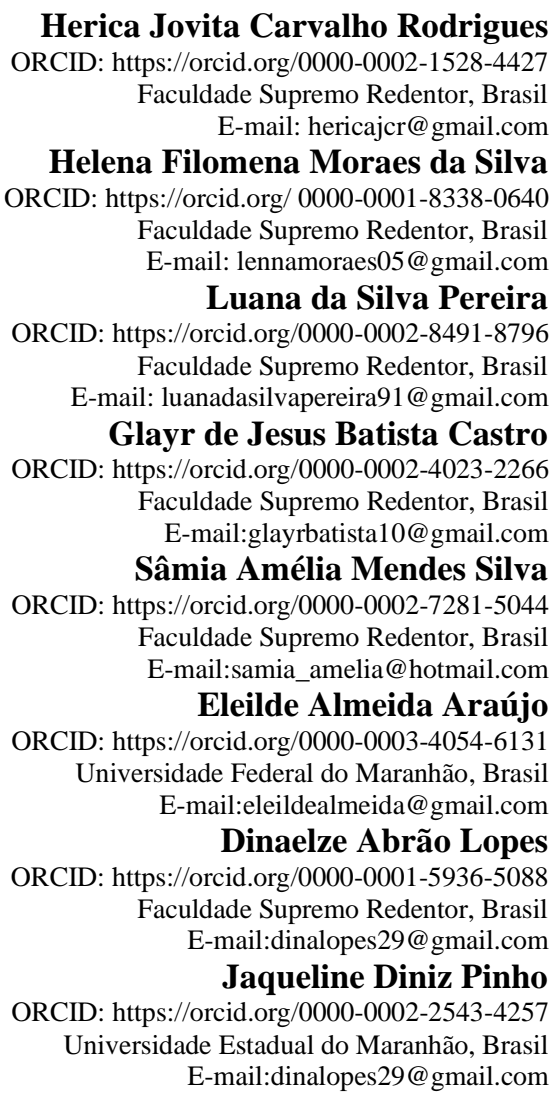

\begin{abstract}
Resumo
As vulvovaginites são as causas mais comuns de corrimento vaginal em mulheres, e as principais consequências associadas estão: infertilidade, parto prematuro ou recém-nascido de baixo peso, aborto, endometrite pós-cesárea, aumento do risco de contrair doenças de transmissão sexual, como a AIDS, gonorréia, tricomoníase, entre outras. Neste contexto, o objetivo deste trabalho foi identificar a prevalência de vulvovaginites em mulheres lavradoras atendidas em uma Unidade Básica de Saúde (UBS) oriundas da zona rural do interior do Maranhão. Para isso, foi realizado um estudo retrospectivo, transversal e descritivo, realizado com mulheres que buscaram atendimento em uma UBS atendidas durante os anos de 2018 e 2020. De acordo com os dados levantados, predominaram mulheres com mais de 51 anos $(28,57 \%)$, lavradoras $(61 \%)$, que se queixaram de leucorreia, e apresentaram no resultado do exame: Gardnerella, Candida e HPV. Os dados apresentados neste trabalho demonstram como a educação em saúde para as mulheres, em especial para aquelas que possuem baixo acesso aos serviços de saúde, é mandatória. Isso permitirá que a busca por atendimento ginecológico ocorra de forma precoce, visto que alguns dos agentes causadores das vulvovaginites, como o HPV, estão associados a vários tipos de cânceres.
\end{abstract}

Palavras-chave: Vulvovaginites; Educação em saúde; Prevenção; Câncer.

\begin{abstract}
Vulvovaginitis is the most common cause of vaginal discharge in women, and the main associated consequences are: infertility, premature birth or low birth weight, miscarriage, post-cesarean endometritis, increased risk of contracting sexually transmitted diseases, such as AIDS, gonorrhea, trichomoniasis, among others. In this context, the objective of this study was to identify the prevalence of vulvovaginitis in women farmers treated at a Basic Health Unit (BHU) from the rural area of the interior of Maranhão. For this, a retrospective, cross-sectional and descriptive study was
\end{abstract}


carried out with women who sought care at a UBS during the period from September 2019 to August 2020. According to the data collected, women over 51 years old predominated $(28.57 \%)$, farm workers $(61 \%)$, who complained of leucorrhoea, and presented: Gardnerella, Candida and HPV in the test results. The data presented in this work demonstrate how health education for women, especially for those who have low access to health services, is mandatory. This will allow the search for gynecological care to occur early, since some of the causative agents of vulvovaginitis, such as HPV, are associated with various types of cancers

Keywords: Vulvovaginitis; Health education; Prevention; Cancer.

\section{Resumen}

La vulvovaginitis es la causa más común de flujo vaginal en la mujer, y las principales consecuencias asociadas son: infertilidad, parto prematuro o bajo peso al nacer, aborto espontáneo, endometritis poscesárea, mayor riesgo de contraer enfermedades de transmisión sexual, como el SIDA, gonorrea, tricomoniasis, entre otros. En ese contexto, el objetivo de este estudio fue identificar la prevalencia de vulvovaginitis en mujeres agricultoras atendidas en una Unidad Básica de Salud (UBS) del área rural del interior de Maranhão. Para ello, se realizó un estudio retrospectivo, transversal y descriptivo con mujeres que buscaron atención en una UBS durante el período de septiembre de 2019 a agosto de 2020. Según los datos recabados, predominaron las mujeres mayores de 51 años (28,57\%), trabajadores agrícolas (61\%), que se quejaron de leucorrea y tenían Gardnerella, Candida y VPH en los resultados de la prueba. Los datos presentados en este trabajo demuestran cómo la educación en salud para las mujeres, especialmente para aquellas que tienen bajo acceso a los servicios de salud, es obligatoria. Esto permitirá que la búsqueda de atención ginecológica sea temprana, ya que algunos de los agentes causantes de la vulvovaginitis, como el VPH, están asociados a varios tipos de cáncer.

Palabras clave: Vulvovaginitis; Educación para la salud; Prevención; Cáncer.

\section{Introdução}

As vulvovaginite são caracterizadas como uma manifestação inflamatória e/ou infecciosa do trato genital feminino inferior (vulva, vagina e epitélio escamoso do colo do útero - ectocérvice), causadas principalmente por bactérias, fungos ou vírus (Ministério da Saúde, 2015). O quadro clínico varia de acordo com a etiologia e pode apresentar quadro com corrimento vaginal, prurido, dor, irritação local. No entanto, vale ressaltar que muitas infecções genitais podem ser assintomáticas (Cavalcante et al., 2005).

As vulvovaginites ou vaginoses são as causas mais comuns de corrimento vaginal patológico, e consequentemente são as causas mais frequentes das queixas ginecológicas, e constituem em um dos problemas mais rotineiros, sendo mais frequentes as bacterianas. Os patógenos mais comuns em sua maioria são Gardnerella vaginalis, por Cândida albicans e Trichomonas vaginalis, os quais são os principais agentes etiológicos da candidíase vulvovaginal, tricomoníase, por exemplo (Andrade et al., 2014).

Dentre as principais consequências associadas à vaginose estão: infertilidade, parto prematuro ou recém-nascido de baixo peso, aborto, endometrite pós-cesárea, aumento do risco de contrair doenças de transmissão sexual, como a AIDS, gonorréia, tricomoníase, entre outras (Frighetto et al., 2016).

Os dados epidemiológicos revelam que as vulvolvaginites é a causa mais frequente de mulheres com queixa de corrimento vaginal. Além disso aquelas mulheres que não relatam sintomas, as vulvovaginites podem representar cerca de $10 \%$ a 30\% (Alves et al., 2021). As mais acometidas são aquelas que possuem baixa renda, e consequentemente dificuldade no acesso aos serviços básicos de saúde (Carvalho \& Brito, 2005). No Estado do Maranhão são escassos os levantamentos de casos, assim como a caracterização dos tipos de agentes etiológicos que ocasionam essa patologia, em especial em mulheres da zona rural. Neste contexto, o objetivo deste foi caracterizar e identificar a prevalência de vulvovaginites em mulheres lavradoras atendidas em uma UBS da zona rural do Estado do Maranhão.

\section{Metodologia}

Esta pesquisa trata-se de um estudo retrospectivo, transversal e descritivo, conforme descrito por Hochman et al (2005). As mulheres que participaram desta pesquisa buscaram atendimento em uma UBS no município de São Bento-MA, 
durante os anos de 2018 e 2020. O município de São Bento possui aproximadamente 45.989 habitantes e um Indice de Desenvolvimento Humano Municipal de 0.6 (IBGE, 2020). O serviço público de saúde é composto por Equipes de Saúde da Família e uma Unidade Básica de Saúde que exclusivamente possui atendimento ginecológico.

Os dados foram obtidos através dos prontuários e fichas disponibilizados nas UBS, os quais foram preenchidos por um profissional de saúde. Dentre as informações que constavam no prontuário, foram coletadas as seguintes informações: idade, estado civil, profissão, gestações, quantidade de partos, principais queixas e resultado dos exames realizados a fim de conhecer o tipo de patógeno presente. Os dados foram tabulados no Excel (Microsoft Office 2013) e analisados no SPSS V22 (IBM Corp., Armonk, NY, EUA). Análises de frequência relativa e absoluta foram realizadas para verificar a distribuição das variáveis categóricas. Os dados foram apresentados através de porcentagens em tabelas.

Esta pesquisa foi submetida ao Comitê de Ética em Pesquisa da Universidade Estadual do Maranhão com aprovação sob $n^{\circ} 5.130 .140$.

\section{Resultados e Discussão (pode ser separado ou junto) (fonte TNR 12 - alinhado esquerda)}

Nesta pesquisa foram coletadas informações de 105 mulheres. Predominou mulheres com mais de 51 anos $(28,57 \%)$, lavradoras (61\%), solteira (51,4\%), que já tiveram mais de 4 partos $(34,2 \%)$ (Tabela 1$)$.

Tabela 1. Caracterização epidemiológica de mulheres da zona rural que buscaram atendimento ginecológico em uma UBS.

\begin{tabular}{|c|c|c|}
\hline VARIÁVEIS & & $\mathbf{N}(\%)$ \\
\hline \multirow{5}{*}{ FAIXA ETÁRIA } & 10-20 anos & $5(4,76)$ \\
\hline & 21- 30 anos & $19(18,09)$ \\
\hline & 31- 40 anos & $27(25,71)$ \\
\hline & 41- 50 anos & $24(22,85)$ \\
\hline & mais de 51 anos & $30(28,57)$ \\
\hline \multirow{5}{*}{ PROFISSÃO } & Lavradora & $61(58,1)$ \\
\hline & Pescadora & $11(10,5)$ \\
\hline & Funcionária Pública & $3(2,9)$ \\
\hline & Estudantes & $3(2,9)$ \\
\hline & Outras profissões & $27(25,6)$ \\
\hline \multirow{3}{*}{ TABAGISMO } & Sim & $2(1,9)$ \\
\hline & Não & $100(95,2)$ \\
\hline & Ex-tabagista & $3(2,8)$ \\
\hline \multirow{5}{*}{ ESTADO CÍVIL } & Casada & $38(36,1)$ \\
\hline & Solteira & $54(51,4)$ \\
\hline & Viúva & $6(5,7)$ \\
\hline & União Estável & $1(0,9)$ \\
\hline & Não Informado & $6(5,7)$ \\
\hline
\end{tabular}




\begin{tabular}{|c|c|c|}
\hline \multirow{5}{*}{ No DE GESTAÇÕES } & 0 & $14(13,3)$ \\
\hline & 1 & $13(12,3)$ \\
\hline & 2 & $26(24,7)$ \\
\hline & 3 & $16(15,2)$ \\
\hline & $>4$ & $36(34,2)$ \\
\hline \multirow{5}{*}{$\mathbf{N}^{\circ}$ DE PARTO } & 0 & $14(13,3)$ \\
\hline & 1 & $13(12,3)$ \\
\hline & 2 & $32(30,4)$ \\
\hline & 3 & $14(13,3)$ \\
\hline & $>4$ & $32(30,2)$ \\
\hline \multirow[t]{3}{*}{ TIPO DE PARTO } & Normal & $75(71,4)$ \\
\hline & Cesárea & $28(26,6)$ \\
\hline & Não Informado & $2(1,9)$ \\
\hline
\end{tabular}

Fonte: Autores (2022).

As principais queixas relatadas pelas mulheres foi a presença de leucorreia, em especial a leucorreia fétida (11,4\%) e leucorréia com pruridos $(10,4 \%)$. As mulheres que relataram leucorreia com prurido também queixavam-se de coceiras (Tabela 2).

Tabela 2. Principais queixas apresentadas pelas mulheres que buscaram atendimento em uma UBS.

\begin{tabular}{ccc}
\hline Queixa Principal & Características & $\mathbf{N}(\%)$ \\
\hline Leucorreia & Leucorreia fétida & $12(11,4)$ \\
\cline { 2 - 3 } & Leucorreia com prurido & $11(10,4)$ \\
\cline { 2 - 3 } & Leucorreia por episódios & $3(2,8)$ \\
\cline { 2 - 3 } & Leucorreia leve & $2(1,9)$ \\
\hline Prurido & Coceira & $3(2,8)$ \\
\cline { 2 - 3 } & Não Informado & $74(70,4)$ \\
\hline
\end{tabular}

Fonte: Autores (2022).

Quanto ao resultado dos exames 39\% apresentavam lactobacilos, 14,2\% Gardnerella e 13,3\% Candida (Tabela 3). Dentre essas mulheres, 14 foram diagnosticas com lesão intraepitelial, com maior prevalência naquelas que apresentavam a infecção pelo HPV (n=9), quatro apresentavam lesão de alto grau e 5 com lesão de baixo grau. 
Tabela 3. Tipos de patógenos identificados nos exames realizados pelas pacientes.

\begin{tabular}{ccc}
\hline Presença de Patógenos & $\mathbf{N}^{\mathbf{0}}$ & $\%$ \\
\hline Candida & 7 & 6,6 \\
Candida/HPV & 1 & 0,9 \\
Cocos & 14 & 13,3 \\
Cocos/Lactobacillos & 1 & 0,9 \\
Gardnerella & 15 & 14,2 \\
Gardnerella/Lactobacillos & 3 & 2,8 \\
HPV & 7 & 6,6 \\
HPV/Lactobacillos & 1 & 0,9 \\
Lactobacillos & 41 & 39 \\
Lactobacillos/Candida & 14 & 13,3 \\
Lactobacillos/HPV/Gardnerella & 1 & 0,9
\end{tabular}

Fonte: Autores (2022).

\section{Discussão}

No presente trabalho, a maioria das mulheres que buscaram atendimento eram lavradoras (61\%). Estas mulheres de baixa renda geralmente possuem dificuldade no acesso aos serviços de saúde, além disso, muitas não buscam tratamento de forma precoce, devido ao medo do exame e/ou do resultado (Almeida et al., 2013). Associado a baixa instrução, está o desconhecimento sobre as consequências da ausência da busca por atendimento ginecológico de forma precoce, o que inviabiliza o rastreamento do câncer de colo de útero (Alves et al., 2016).

As mulheres desta pesquisa relataram como principal queixa a leucorreia, também conhecida como corrimento vaginal. O corrimento vaginal é uma das preocupações mais frequentes entre as mulheres na idade reprodutiva, sendo uma das principais causas para esta sintomatologia a presença de patógenos causadores de vulvovaginite (Carvalho et al., 2021).

Alguns patógenos que causam as vulvovaginites se enquadram no grupo das Infecções Sexualmente Transmissíveis, dessa forma, as vulvovaginites devem ser encaradas como um problema de saúde pública, uma vez que podem desencadear outras patologias. Diante do exposto, deve-se considerar esse agravo como uma das prioridades na atenção à saúde da mulher de modo a oferecer prevenção, identificação e tratamento oportuno levando em consideração cada especificidade do acometimento (Tabile et al., 2016)

Quanto ao relato de algumas mulheres desta pesquisa estarem grávidas no momento do exame, é necessário ressaltar que a vulvovaginite em mulheres grávidas, pode gerar complicações mais graves como: crescimento intrauterino restrito, parto prematuro, e/ou a mulher pode sofrer aborto, ou ficar suscetível a infecções pela imunodeficiência humana (HIV), vírus da hepatite B (HBV) entre outros. O recém-nascido, por sua vez, pode apresentar baixo peso ou infecções puerperais (Nunes $e t$ al., 2018; Carvalho et al., 2021). Devido, a isso, faz-se necessário haver uma política de educação em saúde com estas mulheres que possuem baixo acesso aos serviços de saúde, para que ocorrências como estas não sejam frequentes.

Em relação aos patógenos identificados os mais prevalentes foram Gardnerella vaginallis $(14,2 \%)$ e Candida sp. (13,3\%), o que denota um desequilibrio na flora vaginal. Gardnerella vaginalis e Candida $s p$ são responsáveis por quase 90\% das vaginites, além de serem consideradas como importantes fatores de risco para o câncer de colo do útero. Acredita-se que as atividades inflamatórias causadas por estes agentes infeciosos, podem gerar alterações no colo uterino como colo avermelhado, hiperemiado e sangramento, deixando o colo de útero mais sensível, podendo ser cofatores de infecção pelo HPV (Scwebke $e t$ al., 2014). 
Dados obtidos em estudos semelhantes a esse encontraram como patógeno mais prevalente nas vulvovaginites a Candida sp seguida da Gardnerella vaginalis (Andrade et al., 2014; Tabille et al., 2016). O Ministério da Saúde discute que as principais vulvoginites são candidíase, vaginose bacteriana e tricomoníase (Ministério da Saúde, 2016). Entretanto essa pesquisa encontrou a Gardnerella vaginalis como patógeno mais prevalente demonstrando que existem diferenciações na prevalência das afecções em diferentes localidades do país.

A candidíase estar associada a diferentes espécies do gênero Candida, esta infecção representa a segunda causa de vaginite aguda, seguido da vaginose bacteriana. Estima-se que a candidiase afeta cerca de $75 \%$ das mulheres em algum momento de sua vida, em especial na fase reprodutiva, e caracteriza-se clinicamente pelo prurido vulvar intenso, ardência, leucorreia, dispareunia, disúria, edema e eritema vulvovaginal (Holanda et al., 2007).

Gardnerella vaginalis possui os fatores de virulência necessários para aderir ao epitélio vaginal do hospedeiro e competir com sucesso com a microbiota vaginal normal pela dominância. A infecção por $G$. vaginalis resulta em aumento do pH e diminuição do potencial de redução-oxidação (redox), contribuindo com o aumento do crescimento de anaeróbios do hospedeiro e supressão de lactobacilos. Ainda mais, estudos reforçam a transmissão sexual de G. vaginalis (Silva Duarte et al., 2019).

Um outro agente infeccioso presente foi o HPV, este vírus está associado a uma porcentagem significativa de carcinomas: a exemplo do câncer de colo de útero (Sabeena et al., 2016), câncer anal (Benevolo et al., 2016), vulvar (Maia $e t$ al., 2013), de cabeça e pescoço (Dok \& Nuytz, 2016), orofaringe (Cleary et al., 2016) e do pênis (Martins et al., 2018). Em câncer de cólon de útero o HPV está envolvido em cerca de até 99,7\% dos casos (Okunade, 2020). Portanto, a disseminação de informação sobre a forma de transmissão, tipos de diagnóstico e medidas profiláticas do HPV, e a importância da adesão a vacinação é importante para que a incidência das neoplasias malignas relacionadas à infecção por HPV diminua, em especial o câncer de colo de útero, que é a segunda maior causa de câncer entre mulheres (Silveira et al., 2006).

A deteç̧ão e o tratamento precoces das vulvovaginites são primordiais, uma vez que evitam outras patologias (Tabille et al., 2016). Além disso, deve-se reconhecer a importância da iniciativa governamental através da realização de campanhas locais que atinjam principalmente as mulheres de baixa renda, bem como a realização de exames preventivos e disponibilização de insumos necessário ao atendimento qualificado dessas mulheres, promovendo acesso facilitado de modo que a adesão ao exame Papanicolau seja maior auxiliando na prevenção de afecções ginecológicas e sobretudo, no câncer do colo do útero que é função primária desse exame.

Vale ressaltar que destacamos o exame Papanicolau como forma de diagnóstico dos agentes microbiológicos, pois este é o único exame disponível nesta unidade às mulheres que chegam com queixas condizentes a vulvovaginites, já que este é um exame específico para rastreio de células neoplásicas cervicais, mas que pode ser usado em serviços onde não dispõe do exame a fresco com microscópio o que se configura como a realidade de todas as unidades de saúde do SUS da referida localidade.

\section{Conclusão}

Os dados apresentados neste trabalho, demonstram que a maioria das mulheres atendidas são lavradoras, que tiveram como principal queixa a leucorreia, e que estavam infectadas pela Gardnerella. Estes dados demonstram a importância de educação em saúde, para que a busca por atendimento ginecológico aconteça de forma precoce, visto que alguns dos agentes causadores das vulvovaginites, como o HPV, estão associados ao câncer de colo do útero. Destacamos a importância de ações e pesquisas voltadas para todas as mulheres, em especial as de baixa renda, nos serviços públicos de saúde. Faz-se necessário novos estudos que colaborem com a comunidade científica com o intuito de entender a patologia e as vulnerabilidades associadas a ela e apontem o caminho para modificar as práticas profissionais. 


\section{Referências}

Almeida, M. S., Barbosa, F. H. F., \& Gomes, M. S. (2013). Prevalência de microrganismos patogênicos presentes em secreções vaginais de pacientes atendidas na Unidade de Saúde de Mazagao-AP/Brasil no Ano de 2009 E 2010. Revista de Biologia e Ciências da Terra, 13. http://joaootavio.com.br/bioterra/workspace/uploads/artigos/747-2809-1-pb-53df93f9cabf1.pdf

Alves, G. B., Alvim, M. C. T. \& Odorizzi V. F., \& Baptista A. B. (2021). Perfil etiológico e epidemiológico das vulvovaginites que acometem mulheres em uma cidade do estado de Tocantins. Revista Eletrônica Acervo Saúde ,13(2): e5383. https://doi.org/10.25248/reas.e5383.2021.

Alves, J., Oliveira W., Mendonça B., Oliveira V., Nogueira D., Barros E., Mota R, Monteiro B., Gonçalves V., \& Guimarães S. (2016). Exame colpocitológico (papanicolau): O conhecimento das mulheres sobre o preventivo no combate do câncer de colo do útero. Revista Faculdade Montes Belos (FMB), 9:125-141

Andrade, S. C., da Silva, F. M. C., Oliveira, S. H. S. \& Leite, K. N. S., da Costa, T. F., \& Zaccara, A. A. L. (2014). Agentes Microbiológicos de vulvovaginites identificados pelo Papanicolau. Revista de Enfermagem UFPE online, 8:233-9. https://doi.org/10.5205/1981-8963-v8i2a9679p338-345-2014

Carvalho, N.S., Eleutério Júnior, J., Travassos, A.G., Santana, L. B. \& Miranda, A. E. (2021). Protocolo Brasileiro para Infecções Sexualmente Transmissíveis 2020: infecções que causam corrimento vaginal. Epidemiologia e Serviços de Saúde. https://doi.org/10.1590/S1679-4974202100007.

Carvalho, J. M. \& Brito F (2005). A demografia brasileira e o declínio da fecundidade no Brasil: contribuições, equívocos e silêncios. Revista Brasileira de Estudos Populacionais, 22(2):351-370. doi.org/10.1590/S0102-30982005000200011.

Cavalcante, V. L. N., Vânia, L. N. \& Miranda A. (2005). Rastreamento de Candidose Vaginal Durante a Prevenção do Câncer Cérvico-Uterino. DST Jornal Brasileiro de Doenças Sexualmente Transmissiveis, 17(1): 44-48. http://www.dst.uff.br/revista17-1-2005/rastreamentodecandidose.pdf

Cleary, C., Leeman L, Higginson D., Katabi N., Sherman E., Morris L., McBride S., Lee N., \& Riaz N (2016) Biological Features of Human Papillomavirusrelated Head and Neck Cancers Contributing to Improved Response. Clinical Oncology in press, 28(7):467-74. 10.1016/j.clon.2016.03.001.

Dok R \& Nuyts S (2016) HPVPositive Head and Neck Cancers: Molecular Pathogenesis and Evolving Treatment Strategies. Cancers 29;8(4). $10.3390 /$ cancers 8040041

Amaral A., Frighetto M., \& Satin N. (2016). Incidência de Gardnerella vaginalis nas amostras de secreção vaginal em mulheres atendidas pelo laboratório municipal de Fraiburgo. Anuário Pesquisa e Extensão Unoesc Videira. https://portalperiodicos.unoesc.edu.br/apeuv/article/view/12246/6461

Hochman B., Xerfan F., Filho R., \& Ferreira L (2005). Desenhos de pesquisa. Acta Cirúrgica Brasileira. https://doi.org/10.1590/S0102-86502005000800002

Holanda A, Fernandes A, Bezerra C, Ferreira M., Holanda M., Holanda J., \& Milão E. (2007). Vulvovaginal candidiasis: symptomatology, risk factors and concomitant anal colonization.Revista Brasileira de Ginecologia e Obstetricia, 29(1):3-9. https://doi.org/10.1590/S0100-72032007000100002

Instituto Brasileiro de Geografia e Estatística (2020). Cidades e Estados: São Bento. https://www.ibge.gov.br/cidades-e-estados/ma/sao-bento.html

Maia B., Mourão A., Rodrigues L., Malheiros C., Baiocchi G., Stiepcich M., Puga R., Lima L., Soares F., \& Rocha R. (2013) MicroRNA Portraits in Human Vulvar Carcinoma. Cancer Prevention Research, 6(11) 1231- 1241. 10.1158/1940-6207.CAPR-13-0121

Martins V., Pinho J., Teixeira A., Nogueira J., Silva F., Maulen V., Khayat A, Calixto J., Costa H., Ramalho L. \& Silva G. (2018). P16INK4a expression in patients with penile cancer. PloS One 13(10):e0205350. 10.1371/journal.pone.0205350

Ministério da Saúde (2015) Protocolo Clínico diretrizes terapêutica e atenção integral a pessoas com infecções sexualmente transmissíveis https://bvsms.saude.gov.br/bvs/publicacoes/protocolo_clinico_diretrizes_terapeutica_atencao_integral_pessoas_infeccoes_sexualmente_transmissiveis.pdf.

Ministério da Saúde (2016). Diretrizes brasileiras para o rastreamento do câncer do colo do útero / Instituto Nacional de Câncer José Alencar Gomes da Silva. Coordenação de Prevenção e Vigilância. Divisão de Detecção Precoce e Apoio à Organização de Rede. - 2. ed. rev. atual. - Rio de Janeiro: INCA, 2016.

Muzny, C. A. \& Schwevke, J. R. (2013) Gardnerella vaginalis: ainda é o principal suspeito na patogênese da vaginose bacteriana, Relatórios Current Infections Disease, 15: 130-135.

Nunes R., França C. \& Traebert, J. (2018). Prevalência de Vulvovaginites na Gestação e sua Associação Com Complicações Perinatais. Arquivos Catarinenses de Medicina, 47:121-132.http://www.acm.org.br/acm/seer/index.php/arquivos/article/view/293

Okunade, K. S. (2020). Human Papillomavirus and Cervical Cancer. Journal of Obstetrict and Gynaecology, 40 (5): 602-608. $10.1080 / 01443615.2019 .1634030$

Rodrigues M., Simões L. \& Diniz C. (2009). Clinical, microbiological and therapeutic aspects of vulvovaginal candidiasis and recurrent vulvovaginal candidiasis: importance of regional surveys. HU Revista, 35(3):175-81

Sabeena, S., Bhat P., Kamath V., Mateus M., Aswathyraj S., Devadiga S., Prabhu S., Hindu M., Chameetachal A., Krishnan A. \& Arunkumar G. (2016) Detection of Genital HPV Infection Using Urine Samples: a Population Based Study in India. Asian Pacific Journal of Cancer Prevention, 17(3):1083-1088. 10.7314/apjcp.2016.17.3.1083

Duarte S., Lima F., Sampaio J, Maia T., Guimarães G., Martins M. \& Depra L. (2019). Fisiopatologia, diagnóstico e tratamento da vaginose bacteriana. Brazilian Journal of Development, 5 (10); 21467- 2175. 10.34117/bjdv5n10

Scwebke J., Muzny C. \& Josey W. (2014). Role of Gardnerella vaginalis in the Pathogenesis of Bacterial Vaginosis: A Conceptual Model. The Journal of Infectious Diseases ,210 (3): 338-343, https://doi.org/10.1093/infdis/jiu089

Tabile P., Lucena H., Chaves J., Fischborn J. \& Jucá R. (2016) Características clínicas, prevalência e diagnóstico de vulvovaginites em ambulatório do interior do Rio Grande do Sul. Revista de Saúde e Ciências Biológicas, 4(3):160-165. 10.12662/2317-3076jhbs.v4i3.657 